\title{
Steering away from a culture of blame
}

Patient Safety in Emergency Medicine

Pat Croskerry, Karen S. Cosby,

Stephen M. Schenkel, Robert Wears

Lippincott Williams \& Wilkins; 2009.

428 pp $\$ 99.95$

$\mathrm{O}$

$\mathrm{n}$ my last shift in the emergency department, I found myself diverted from a task and then rediverted from my diversion seven times within 10 minutes. As I stood there holding five charts in my hands, trying to determine what I was going to be doing with each of those patients, it was very clear to me how error-prone an emergency department is.

For that reason, a book such as this, which aims to reduce errors in the delivery of emergency care, is most welcome and important.

The book begins with a retrospective on sources of errors and a history of error management, then turns to a prospective viewpoint and considers issues around designing safe processes for emergency departments.

This textbook is rich in content and delves deeply into safety and error management issues both from the emergency and the non-emergency worlds. However, some of this may be beyond the needs of the average reader. For example, the sections on

\section{We are an "inquest culture," waiting for}

\section{errors to occur.}

the history of error management are fascinating, but if the reader has limited time, some of these sections might benefit from labelling as background material as opposed to core content. That said, the book is very well organized and the chapters can stand alone. As a result, readers who have specific questions can select only the sections or chapters relevant

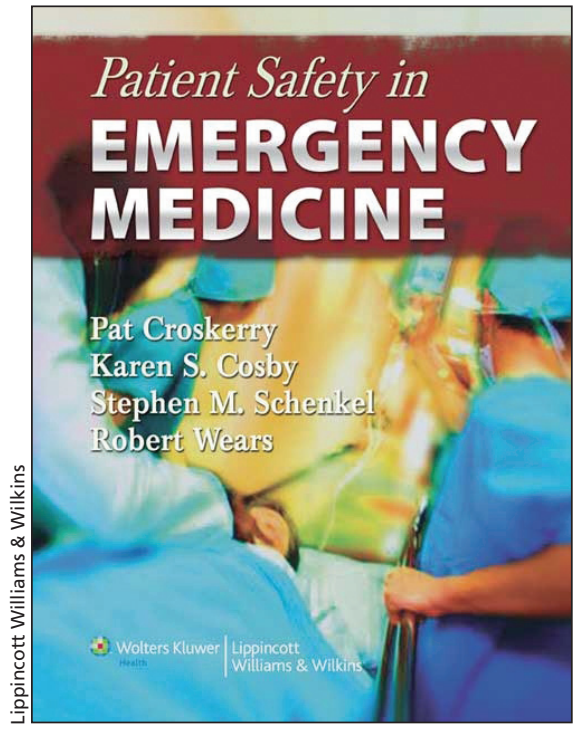

to them and still come away with some practical guidance.

In addition to chapters in the book regarding specific emergency department functions (medication delivery, discharges, radiology, etc.), there is a brief and intriguing mention of skills. The authors suggest adding training in how to function in (and improve the function of) the emergency department to the existing training curriculum, a theme that is expanded on later in the text under the heading "Optimizing Human Perfor-

mance." As the authors point out, given that the frequent error sources are known, it makes perfect sense to train residents in how to avoid them.

Readers familiar with managerial leadership research or those who have been in management roles will recognize some of the models and tools quoted by the authors, but they may still learn from how these are applied to improving physician's operational error management and prevention in the emergency department.

To their credit, the authors clearly state that while new processes, such as the adoption of electronic health records, may decrease some sources of error, such changes also hold the possibility of new errors.

Occasionally, mention is made of the political importance of emergency departments, of the public expectations of shorter wait times and the higher media profile given to errors in emergency care. The elephant in the room that the authors allude to repeatedly, but wisely and understandably do not address directly, is the need for systemwide political resolution of emergency department overcrowding. This is, without a doubt, a factor that contributes to errors, morbidity and mortality. A glance across the Atlantic to the United Kingdom (its health care system is cited frequently in the text) provides an example of a health care system that managed to reduce its wait times dramatically, but only after direct political intervention.

These concerns are common to emergency departments in developed nations, many of which, like Canada, have government involvement in delivering health care. It is unfortunate that the taxonomy and analysis provided by the authors does not go far or wide enough to include Canadian provincial and territorial governments or international governments.

It has been said that we are an "inquest culture," waiting for errors to occur and then voraciously looking for whom to blame. This book provides the tools to steer clear of many of the common pitfalls and away from the culture of blame toward a healthier approach to error prevention.

\section{Daniel Kollek MD}

Associate professor

Emergency Medicine

McMaster University

Hamilton, Ont. 